\title{
Effect of Autofrettage in the Thick-Walled Cylinder with a Radial Cross-Bore
}

\author{
Hongjun Li, Richard Johnston and Donald Mackenzie \\ Department of Mechanical Engineering \\ University of Strathclyde \\ Glasgow, United Kingdom
}

\begin{abstract}
The effect of autofrettage on the stress level in thick-walled cylinders with a radial crossbore is investigated by applying inelastic Finite Element Analysis (FEA) with cyclic pressure loading. A macro is created in ANSYS to calculate the equivalent alternating stress intensity, $\boldsymbol{S}_{\text {eq, }}$ based on the ASME Boiler and Pressure Vessel Code. The value of $S_{\mathrm{eq}}$ is used to evaluate the fatigue life of the vessel. For a specific cyclic load level, a distinct optimum autofrettage pressure is identified by plotting autofrettage pressure against the number of cycles from design fatigue data. The fatigue life of the autofrettaged vessel with such an optimum pressure is significantly increased compared with the case where no autofrettage is used.
\end{abstract}




\section{Nomenclature}

inner radius

b outer radius

c cross-bore radius

E Young's Modulus

$N_{\mathrm{f}} \quad$ number of cycles to failure

$\boldsymbol{P}_{\text {auto }} \quad$ autofrettage pressure

$\mathrm{S}_{\mathrm{ij}} \quad$ stress differences

$\boldsymbol{S}_{\mathrm{eq}} \quad$ equivalent alternating stress intensity

$\boldsymbol{S}_{\text {alt ij }} \quad$ alternating stress intensity

$\sigma_{\mathrm{n} i \mathrm{j}} \quad$ normal stresses

$\sigma_{\mathrm{nm}} \quad$ mean Stress

$\boldsymbol{\sigma}_{\mathrm{y}} \quad$ yield stress

v Poisson's Ratio 


\section{Figure captions}

Figure 1 Fluctuating Stress Pattern

Figure 2 The thick-walled cylinder with a radial cross-bore

Figure 3 Finite element analysis model

Figure 4 Variation of $\boldsymbol{S}_{\text {eq }}$ with $\boldsymbol{P}_{\text {auto }}, b / a=1.75$ and $c / a=0.2$

Figure 5 Variation of $\boldsymbol{N}_{f}$ with $\boldsymbol{P}_{\text {auto }}, \mathrm{b} / \mathrm{a}=1.75$ and c/a $=0.2$

Figure 6 Variation of $\boldsymbol{S}_{\text {eq }}$ with $\boldsymbol{P}_{\text {auto }}$ for $b / a=1.5$

Figure 7 Variation of $\boldsymbol{S}_{\text {eq }}$ with $\boldsymbol{P}_{\text {auto }}$ for $b / a=1.75$

Figure 8 Variation of $\boldsymbol{S}_{\mathrm{eq}}$ with $\boldsymbol{P}_{\text {auto }}$ for $b / a=2$

Figure 9 Variation of $\boldsymbol{S}_{\text {eq }}$ with $\boldsymbol{P}_{\text {auto }}$ for c/a $=0.05$

Figure 10 Variation of $\boldsymbol{S}_{\text {eq }}$ with $\boldsymbol{P}_{\text {auto }}$ for c/a $=0.1$

Figure 11 Variation of $\boldsymbol{S}_{\text {eq }}$ with $\boldsymbol{P}_{\text {auto }}$ for c/a $=0.15$

Figure 12 Variation of $\boldsymbol{S}_{\text {eq }}$ with $\boldsymbol{P}_{\text {auto }}$ for c/a $=0.2$

Figure 13 Variation of $\boldsymbol{S}_{\text {eq }}$ with $\boldsymbol{P}_{\text {auto }}$ for c/a $=0.25$

Figure 14 Fatigue life comparison for $\mathrm{b} / \mathrm{a}=1.5$

Figure 15 Fatigue life comparison for $\mathrm{b} / \mathrm{a}=1.75$

Figure 16 Fatigue life comparison for $\mathrm{b} / \mathrm{a}=2$

\section{Listing of table}

Table 1 Limit and first yield pressures. 


\section{Introduction}

In the autofrettage process, thick-walled cylinders are partially yielded by means of a hydrostatic pressure. This produces a larger strain at the inner surface of the cylinder, compared to the outer surface, and so it begins to yield from the inner bore. The size of the autofrettage pressure determines the amount of yielding and therefore the amount of the material at the bore that plastically deforms. When the autofrettage pressure is removed, there is residual compressive stress at the bore and residual tensile stress at the outer surface of the cylinder. This residual compressive stress can serve to reduce tensile stress developed as a result of further applied internal pressures. The process of autofrettage is therefore very useful in improving the fatigue life of cylinders because of the reduction in stress.

Texts such as that of Hill [1], Lubliner [2] and Brownell \& Young [3] and design Codes such as ASME B\&PV Code Division 3 Alternative Rules for High Pressure Vessels [4] give the basis for calculating initiation and spread of plastic deformation during loading, the residual stress upon unloading and the "combined" residual plus operating stress when subsequently loaded to operating pressure. However, optimum autofrettage pressures based on thick cylinder theory are of limited applicability in pressure vessel design, as most real cylindrical vessels incorporate stress raising features which increase the complexity of the stress distribution and cause local stress concentration. Cross-holes in the vessel, such as fluid entry/exit bores, bursting cap ports, etc., give rise to complex stress and strain concentration effects in the vicinity of the hole [5-7]. As the cross hole configuration is not amenable to an accurate analytic solution of the autofrettage process, "Numerical elastic-plastic analyses or experimental techniques..." [4] are required to establish the autofrettage residual stresses. In this paper the optimum autofrettage pressure of a range of thick cylinders with cross holes is determined using 3D elasticplastic finite element analysis. The optimum autofrettage pressure is defined here as that leading to optimum fatigue life according to the ASME VIII Div. 3 Article KD-3 Fatigue Evaluation [4] for a design pressure equal to half the limit pressure of the vessel. The 1998 version of the Code is used as this was the current available version when the work reported was initiated [8]. 


\section{Autofrettage and fatigue life}

This theory underlying Article KD-3 Fatigue Evaluation proposes that the fatigue life of a vessel design is controlled by the Alternating Stress Intensity, $S_{a l t}$, and the Associated Mean Stress, $\sigma_{\mathrm{nm}}$, normal to the plane of $\mathrm{S}_{\mathrm{alt}}$. These stresses are combined to define the Equivalent Alternating Stress Intensity, $\boldsymbol{S}_{\mathrm{eq}}$, which is used with design fatigue curves to determine the number of cycles to failure, $\boldsymbol{N}_{\mathrm{f}}$.

The alternating stress intensity, $\boldsymbol{S}_{\text {alt }}$, represents the maximum range of shear stress. The associated mean stress, $\sigma_{\mathrm{nm}}$, is the mean value of stress normal to the plane subjected to maximum alternating stress intensity. These values are demonstrated in Figure 1.

Three principal stresses, $\sigma_{1}, \sigma_{2}, \sigma_{3}$ are used to calculate the stress differences, $\mathrm{S}$, at each pressure.

$$
\begin{aligned}
& S_{12}=\sigma_{1}-\sigma_{2} \\
& S_{23}=\sigma_{2}-\sigma_{3} \\
& S_{31}=\sigma_{3}-\sigma_{1}
\end{aligned}
$$

$\mathrm{S}_{\mathrm{ij}}$ is used to represent any one of these differences. The largest and smallest algebraic stress differences $\left(S_{i j \max }, S_{i j \min }\right)$ for the design cycle were then identified.

The alternating stress intensity is given by:

$$
\mathrm{S}_{\mathrm{alt} \text { ij }}=0.5\left(\mathrm{~S}_{\mathrm{ij} \max }-\mathrm{S}_{\mathrm{ij} \min }\right)
$$

The stresses normal to the plane of the maximum shear stress, associated with the three alternating stress intensities $\left(\mathrm{S}_{\text {alt }}\right.$ ij $)$ are given by:

$$
\begin{aligned}
& \sigma_{\mathrm{n} 12}=0.5\left(\sigma_{1}+\sigma_{2}\right) \\
& \sigma_{\mathrm{n} 23}=0.5\left(\sigma_{2}+\sigma_{3}\right) \\
& \sigma_{\mathrm{n} 31}=0.5\left(\sigma_{3}+\sigma_{1}\right)
\end{aligned}
$$

$\sigma_{\mathrm{n}}$ ij is used to represent any one of the three normal stresses. The largest and smallest normal stresses $\left(\sigma_{\mathrm{n} i \mathrm{j} \max }, \sigma_{\mathrm{n} \text { ij min }}\right)$ for the whole design cycle is identified. The associated mean stress, $\sigma_{\mathrm{nm}}$, was determined by:

$$
\sigma_{\mathrm{nm} \text { ij }}=0.5\left(\sigma_{\mathrm{n} \text { ij } \max }+\sigma_{\mathrm{n} \text { ij min }}\right)
$$


The value of the associated mean stress is limited to a value not less than zero. Using the values obtained for the alternating stress intensity and the associated mean stress, the equivalent alternating stress intensity can be calculated.

$$
S_{\text {eq ij }}=S_{\text {alt ij }}\left(1 /\left(1-\beta \sigma_{n m \text { ij }} / S^{\prime}{ }_{a}\right)\right)
$$

From the above relationship $\mathrm{S}_{\mathrm{a}}$ is the allowable amplitude of the alternating stress component when $\sigma_{\mathrm{nm}}=0$ and $\mathrm{N}=10^{6}$ cycles. This was taken as $131 \mathrm{~N} / \mathrm{mm}^{2}$ from the design fatigue curves. The value of $\beta$ is a constant value of 0.2 . The value of $\beta \sigma_{\mathrm{nm} \text { ij }} / \mathrm{S}_{\mathrm{a}}$, was limited to a maximum of 0.9 if it exceeded this value.

Three values of $\boldsymbol{S}_{\mathrm{eq} \mathrm{ij}}$ are obtained for the design cycle. The largest of these is used in combination with the design fatigue curve to determine the maximum number of cycles to failure. The largest value is used, as it will be the main driver towards failure.

In the autofrettage process, the location of maximum stress may differ at different parts of the cycle, as will the maximum stress ranges, etc. It was therefore necessary to monitor the stress at all nodes in the vicinity of the cross hole, where the significant stresses generally occurred. A macro was written to incorporate all the fatigue calculations at nodal level and give an output of the equivalent alternating stress intensity, $\boldsymbol{S}_{\mathrm{eq}}$.

\section{Modeling and analysis}

The thick cylinder with radial cross-bore is illustrated in Fig. 2. A one-eighth model of the cylinder was constructed in ANSYS 8.1 [9] with three-dimensional SOLID45 elements. Based on the mesh study, the finer mesh was created around the cross-bore where maximum stress occurred, as shown in Fig. 3. There are 20 elements in thickness and circumference direction of the cylinder and 22 elements in the direction of the crossbore's circumference. Symmetry boundary conditions were applied to three surfaces in order to show one-eighth symmetry. The end of the cylinder remote from the cross-bore was constrained in order to keep the plane section flat and an axial thrust was applied simulating closed end conditions. For the size of cross holes and cylinders considered, cross hole end thrust has a negligible effect on limit pressure and stress distribution [7] and is neglected. The internal pressure was applied to the inner surface of the bore as well as the inner surface of the cross-bore. The internal pressure load cycle consists of loading 
from zero to the autofrettage pressure, unloading to zero pressure then reloading to design pressure. The specified design pressure in all cases is half the limit load of the vessel. A range of autofrettage pressures (between yield pressure and limit pressure) was considered for each vessel.

The material chosen for all models was a typical low alloy steel as used in standard pressure vessels. It was assumed to be elastic perfectly plastic in behaviour and follow the Von Mises yield criteria. The Bauschinger effect is not considered. The material properties used are yield stress, $\boldsymbol{\sigma}_{\mathrm{y}}=300 \mathrm{MPa}$, Young's Modulus, $\boldsymbol{E}=200 \mathrm{GPa}$ and Poisson's Ratio, $\boldsymbol{v}=0.3$.

The internal radius of the cylinder was kept constant at $\mathrm{a}=0.01 \mathrm{~m}$. Three cylinder radius ratios, $b / a$, were analyzed

$$
b / a=1.5,1.75,2
$$

and five cross-bore radius ratios, $c / a$, were analyzed for each cylinder radius ratio.

$$
\mathrm{c} / \mathrm{a}=0.05,0.1,0.15,0.2,0.25
$$

The model length is $0.35 \mathrm{~m}$ which ensures that the stress distribution at the end remote from the cross bore has no effect on the stress distribution near the cross-bore.

In order to define the design pressure and the range of possible autofrettage pressures, limit analysis is performed for each model (autofrettage only occurs between the yield and limit pressures, as the cylinder will only plastically deform within these bounds). By loading the cylinder up to autofrettage pressure then unloading it to zero pressure the residual stress distribution can be seen. If the cylinder is then loaded up to design pressure the operating stress distribution is obtained.

For the models where the ratio b/a varies, new autofrettage ranges had to be found, which meant finding a new limit pressure and therefore the design pressure changed. The design pressure was kept specific to the particular geometry of the design (1/2 limit pressure). The limit and first yield pressures for all configurations investigated are listed in table 1. The limit pressures reported are slightly higher than reported by Dixon and Perez [10]. Example is the case of $\mathrm{b} / \mathrm{a}=2$ and $\mathrm{c} / \mathrm{a}=0.2$, the limit pressure is $220 \mathrm{MPa}$, but based on Dixon's Remaining Strength Factor (RSF) the value, the limit would be about $200 \mathrm{MPa}$. 
In the case where the cross-bore size was being investigated there is little difference in the limit pressure and the design pressure doesn't change much as well.

The same analysis was performed for each of the cylinder radius, and cross-bore, ratios when autofrettage was not used. This allowed comparisons to be made between the two situations.

\section{Results}

For the model with $b / a=1.75$ and $c / a=0.2$, the range of autofrettage pressure was found to be between $45 \mathrm{MPa}$ and $176 \mathrm{MPa}$, which leads to a design pressure of $88 \mathrm{MPa}$. The autofrettage pressure, $\boldsymbol{P}_{\text {auto }}$, is plotted against the maximum equivalent alternating stress intensity, $\boldsymbol{S}_{\text {eq }}$, as shown in Figure 4. The curve shows a distinct dip indicating an optimum $\boldsymbol{P}_{\text {auto }}$ of around $115 \mathrm{MPa}$. If the standard design pressure was applied without autofrettage, the resultant value of $\boldsymbol{S}_{\text {eq }}$ would be $177 \mathrm{MPa}$. (This is the ideal elastic analysis case: in practice the vessel would be subject to a hydro-test at 1.25 times design pressure, $110 \mathrm{MPa}$, which would effectively autofrettage the vessel). Comparing with the value of $\boldsymbol{S}_{\text {eq }}(148 \mathrm{MPa})$ at optimum $\boldsymbol{P}_{\text {auto }}$ shows a reduction of $16.4 \%$ in stress through the autofrettage process. It is clear from Code Design Fatigue Curves [7] that the increase in $\boldsymbol{S}_{\mathrm{eq}}$ will result in decrease in the number of cycles to failure. The lowest $\boldsymbol{S}_{\mathrm{eq}}$ at optimum $\boldsymbol{P}_{\text {auto, }}$, therefore, will correspond to a maximum number of cycles to failure as shown in Figure 5. This figure shows the relationship between the numbers of cycles to failure, $\boldsymbol{N}_{\mathrm{f}}$, against $\boldsymbol{P}_{\text {auto }}$ for non-welded parts made from low alloy steel.

\section{Effect of cross-bore ratio}

For each radius ratio there were five cross-bore ratios investigated, the graphs of autofrettage pressure against $\boldsymbol{S}_{\text {eq }}$ were plotted in Figures 6-8 for three different radius ratios. All the curves have the same characteristics and follow the same trends, showing a clear resemblance to previous work done on the effect of autofrettage on plain thickwalled cylinders [3].

There was little change in $S_{\text {eq }}$ if the autofrettage pressure applied is less than the design pressure. As $\boldsymbol{P}_{\text {auto }}$ increases past design pressure, the stress values decrease rapidly to the optimum pressure and then start to rise slowly. The optimum autofrettage pressures for 
all cross-bore ratios were found to be very close to each other. For $\mathrm{R}=1.5$ the optimum $\boldsymbol{P}_{\text {auto }} \mathrm{S}$ are around $85 \mathrm{MPa}$ and in the range of $62.5 \%-69.6 \%$ limit pressures $(1.35-1.39$ design pressures). For $\mathrm{R}=1.75$ the optimum $\boldsymbol{P}_{\text {auto }} \mathrm{S}$ are in the range of $110 \mathrm{MPa}-120 \mathrm{MPa}$, i.e. $60.1 \%-66.7 \%$ limit pressures. For $\mathrm{R}=2$ the optimum $\boldsymbol{P}_{\text {auto }} \mathrm{S}$ are in the range of 140MPa-150MPa, i.e. 60\%-69.4\% limit pressures.

When the autofrettage pressure is above the optimum $\boldsymbol{P}_{\text {auto, }}$, the higher cross-bore ratio is, the higher $\boldsymbol{S}_{\mathrm{eq}}$ indicating shorter fatigue life. The trend is opposite while the autofrettage pressure is below the optimum $\boldsymbol{P}_{\text {auto. }}$. The value of $\boldsymbol{S}_{\mathrm{eq}}$ at optimum pressure increased as the cross-bore ratio was increased. However, this character is not very obvious for larger radius ratio, $b / a=2$.

\section{Effect of radius ratio}

Increasing the radius ratio will increase the limit pressure, design pressure and the range of autofrettage pressure. The effect of radius ratio on $\boldsymbol{S}_{\text {eq }}$ was demonstrated in Figures 913 for each cross-bore ratio. These curves clearly show that a higher radius ratio will result in a higher optimum autofrettage pressure. The value of $\boldsymbol{S}_{\text {eq }}$ at optimum $\boldsymbol{P}_{\text {auto }}$ remains very close to a constant value despite the change in radius ratio. This is true for all cross-bore ratios. The radius ratio will therefore have little effect on the fatigue life. It should be noted that the design pressures for three radius ratios are different in present investigation.

\section{Effect of autofrettage}

The pressure vessel with optimum autofrettage done was compared to that where no autofrettage had taken place. Figure 14-16 show plots of $\boldsymbol{S}_{\text {eq }}$ (Primary Y axis) and fatigue life $\boldsymbol{N}_{\mathrm{f}}$ (Secondary Y axis) against cross-bore ratio for three radius ratios respectively. The fatigue life of the vessel is much greater where autofrettage has been used. This is more apparent at the smallest cross-bore ratio where a difference of around 490,000 cycles to failure can be seen for $b / a=1.5$. This equates to autofrettage improving the fatigue life of cylinder by three times that where autofrettage had not been used. For the autofrettaged cylinder $\mathbf{S}_{\mathrm{eq}}$ increases with increasing cross-bore ratio and the fatigue life of the autofrettaged cylinder decreases. The number of cycles to failure slowly increases in the case where no autofrettage has been used. However, this is not such a clear trend. 
This along with the decreasing fatigue life of the autofrettaged vessel makes the difference become less with increasing cross-bore. Even for the largest cross-bore ratio the fatigue life can be improved by around two times the number of cycles to failure.

\section{Conclusion}

This paper investigated the effects of introducing a radial cross-bore in an autofrettaged thick-walled cylinder on fatigue life according to the Fatigue Evaluation guidelines of ASME Boiler and Pressure Vessel Code. It is concluded that:

1. The cross-bore ratio has little effect on the value of optimum autofrettage pressure, which is mainly determined by the cylinder radius ratio.

2. The fatigue life decreases with increasing cross-bore ratio for a specific cylinder radius ratio.

3. The fatigue life of an optimally autofrettaged cylinder stays constant for a specific cross-bore ratio regardless of change in the cylinder radius ratio.

4. The fatigue life can be greatly improved by using autofrettage, especially for smaller cross-bore ratios.

\section{References}

[1] Hill R, The mathematical theory of plasticity, Oxford University Press, Oxford, 1950

[2] Lubliner J, Plasticity Theory, Macmillan Publishing Company, New York, 1990

[3] Brownell L E \& Young E H, Process Equipment Design, John Wiley \& Sons, New York, ISBN 0-471-11319-0, 1995

[4] ASME Boiler \& Pressure Vessel Code Division 3 Alternative Rules for High Pressure Vessels, The American Society of Mechanical Engineers, New York, 1998.

[5] Fessler, H. and Lewin, B. H., "Stress distribution in a tee junction of thick pipes", British Journal of Applied Physics, 7, 76-79, 1956.

[6] Gerdeen J C. "Analysis of stress concentrations in thick cylinders with side holes and cross holes", J. Eng. for Industry, 94, 815-823, 1972. 
[7] Makulsawatudom P, Mackenzie D \& Hamilton R, "Stress Concentration at Crossholes in Thick Cylindrical Vessels," J. Strain Analysis for Engineering Design, 39, pp471-481, 2004.

[8] Johnston R, “A Finite Element Simulation of Autofrettage in High Pressure Vessels”, BSc. Thesis, University of Strathclyde, Glasgow, 2006.

[9] ANSYS Inc. ANSYS version 8.1, Canonsberg, PA 15317, 2004

[10] Dixon, R.D. and Perez, E. H., "Effects of Cross-bores on the Limit Load of High Pressure Cylindrical Vessels", High Pressure Technology, ASME PVP, Vol. 371, pp. 119-123, 1998 
Figures

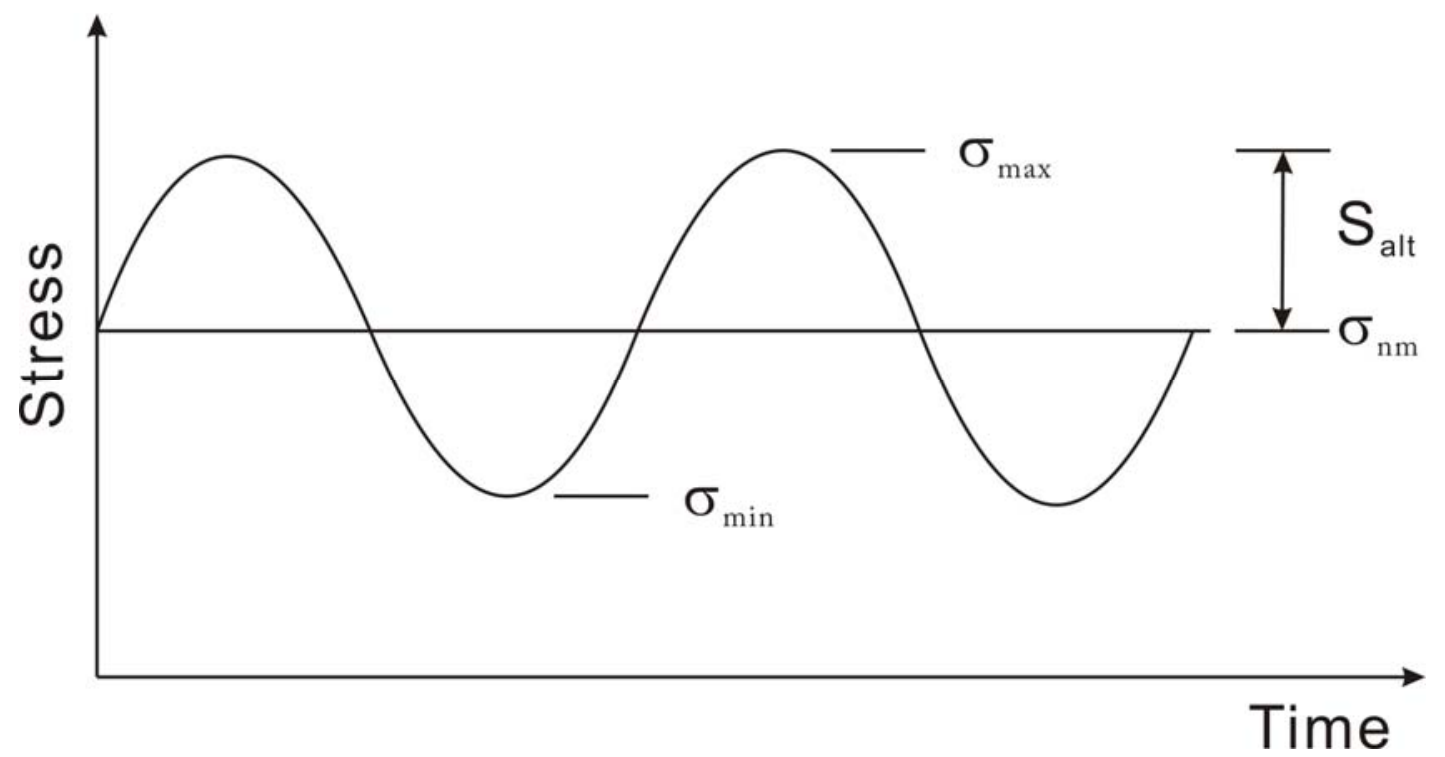

Figure 1 Fluctuating Stress Pattern 


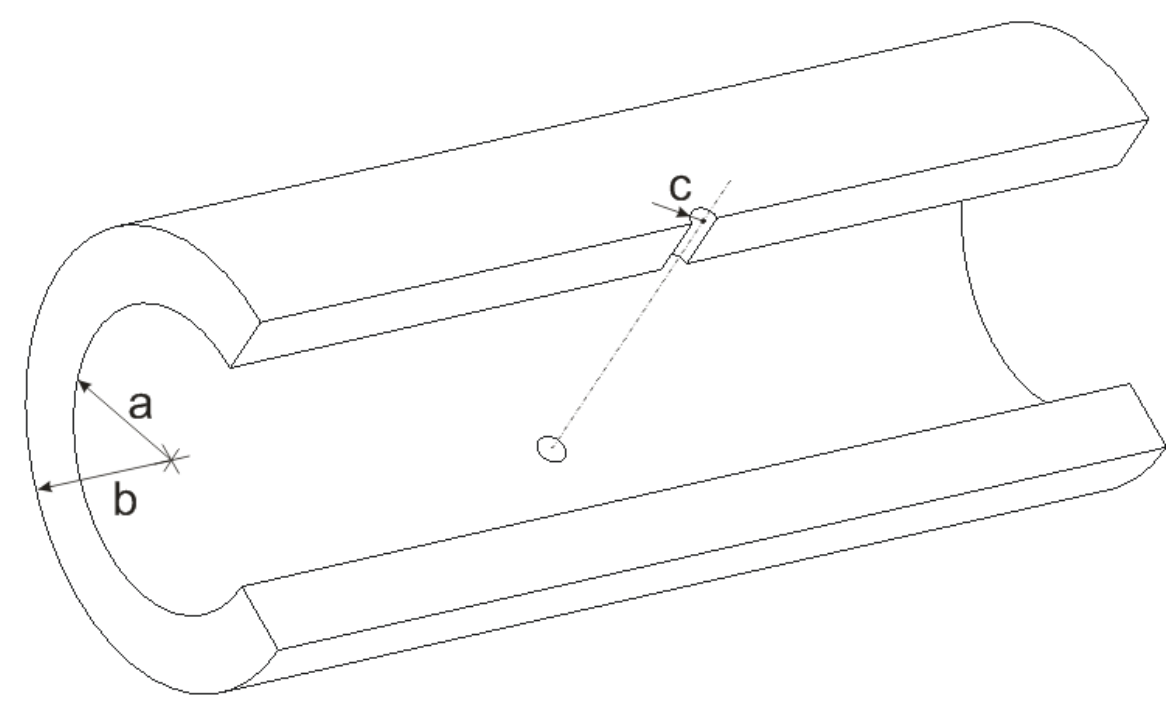

Figure 2 The thick-walled cylinder with a radial cross-bore 

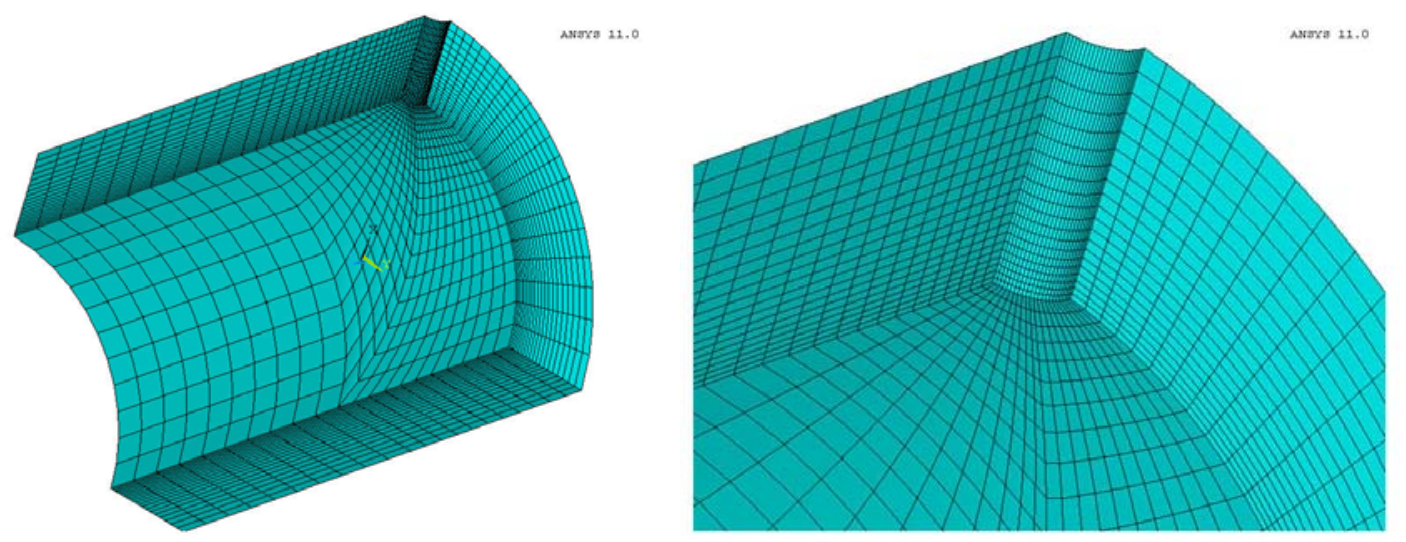

Figure 3 Finite element analysis model 


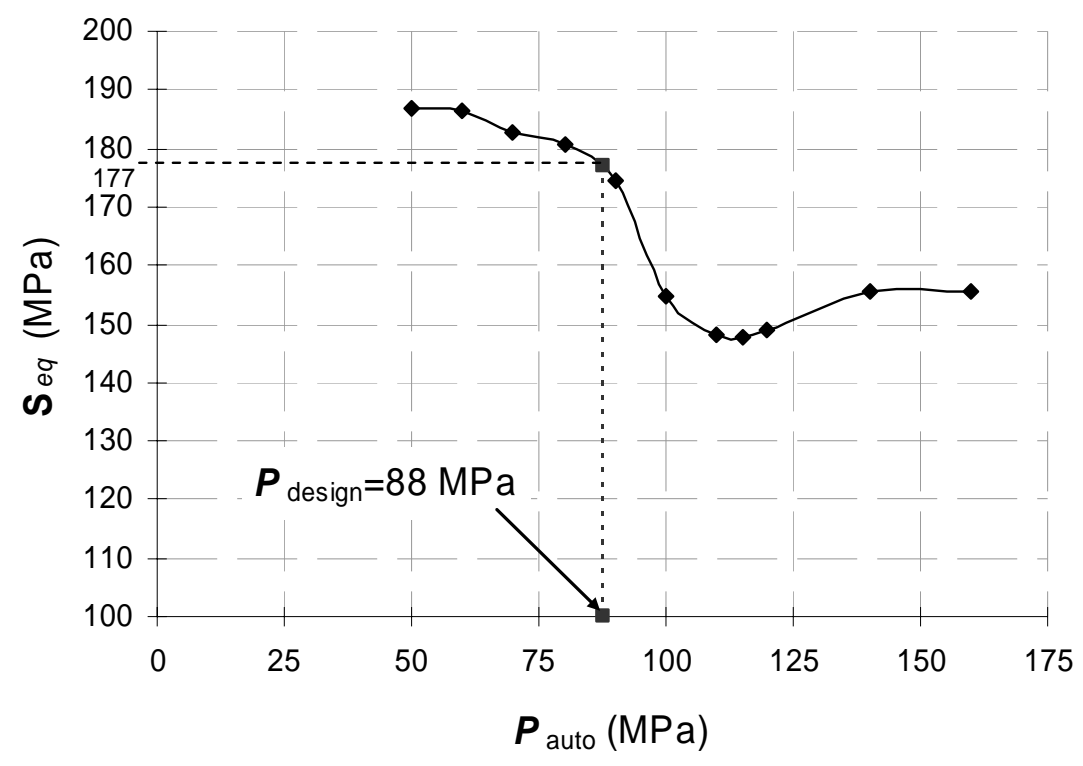

Figure 4 Variation of $\boldsymbol{S}_{\mathrm{eq}}$ with $\boldsymbol{P}_{\text {auto }}, b / a=1.75$ and $c / a=0.2$

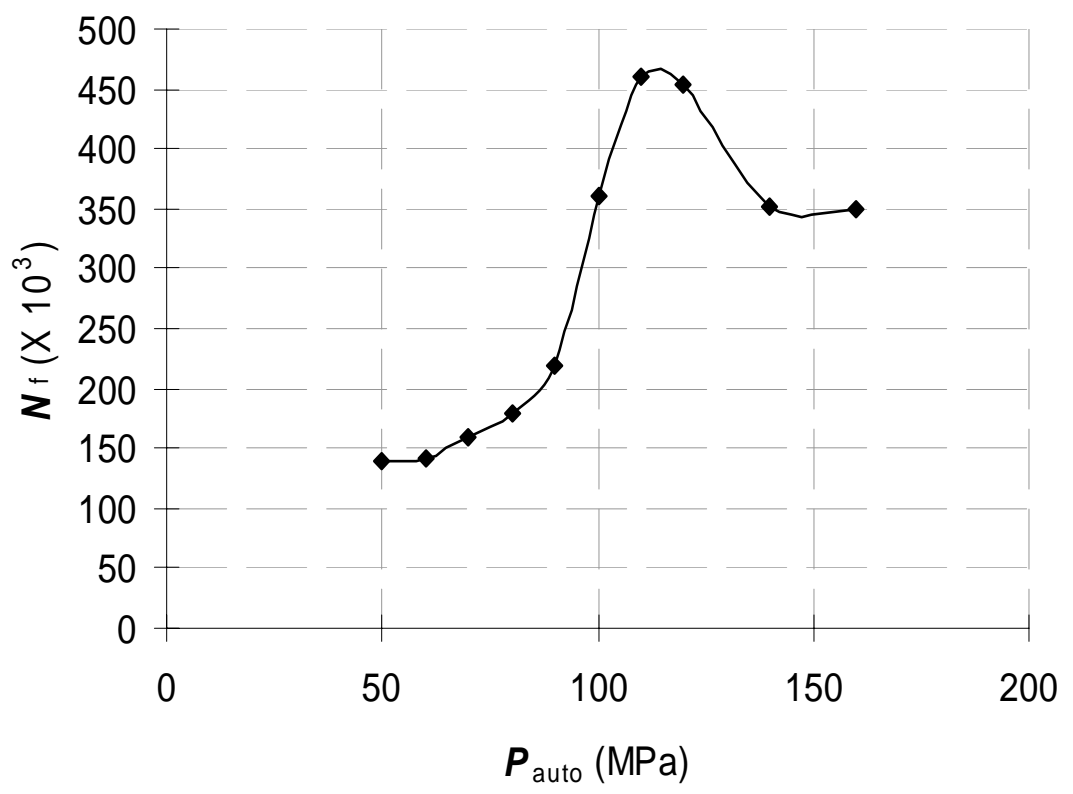

Figure 5 Variation of $\boldsymbol{N}_{\mathrm{f}}$ with $\boldsymbol{P}_{\text {auto }}, \mathrm{b} / \mathrm{a}=1.75$ and c/a=0.2 


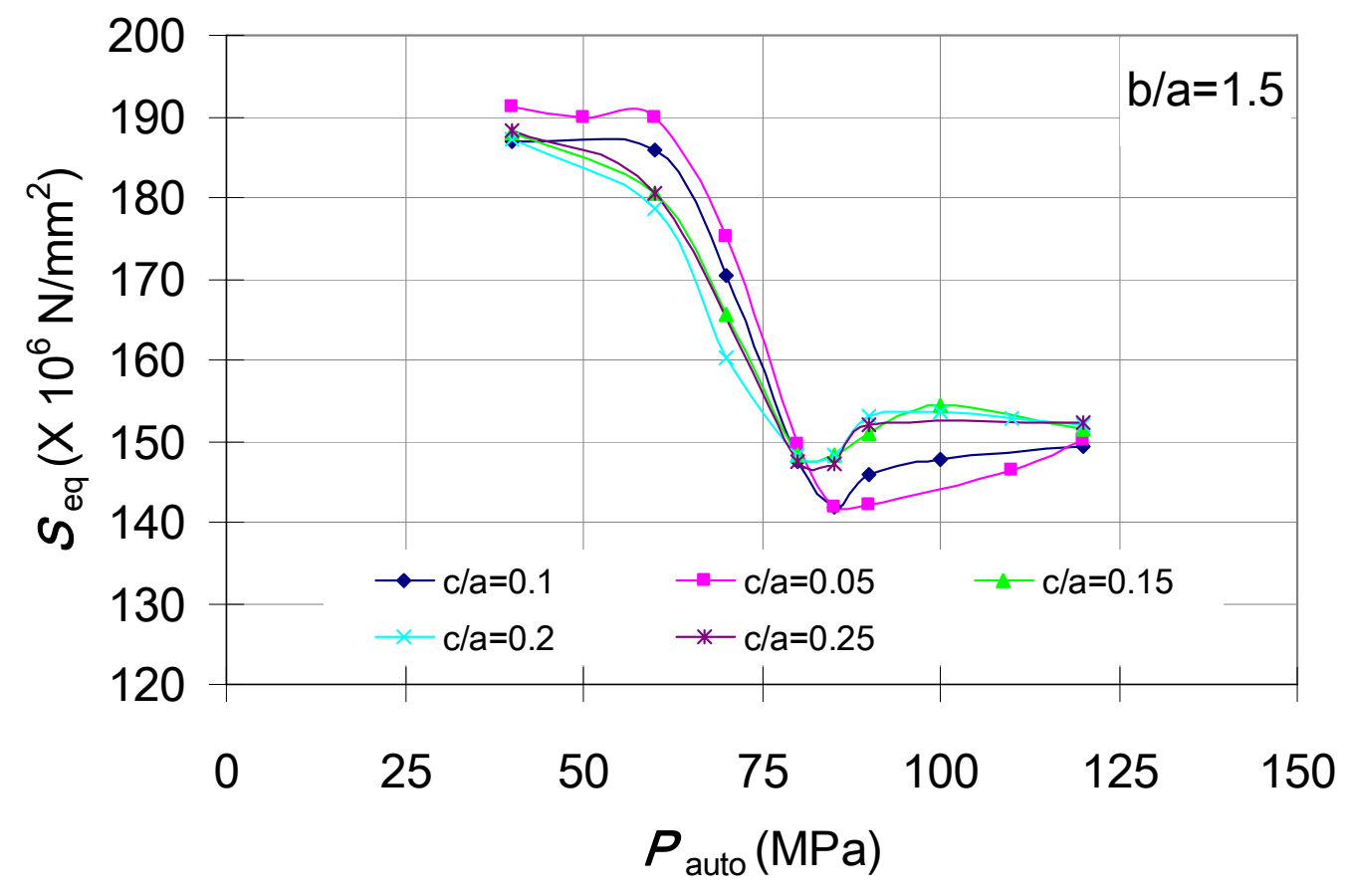

Figure 6 Variation of $\boldsymbol{S}_{\text {eq }}$ with $\boldsymbol{P}_{\text {auto }}$ for $b / a=1.5$

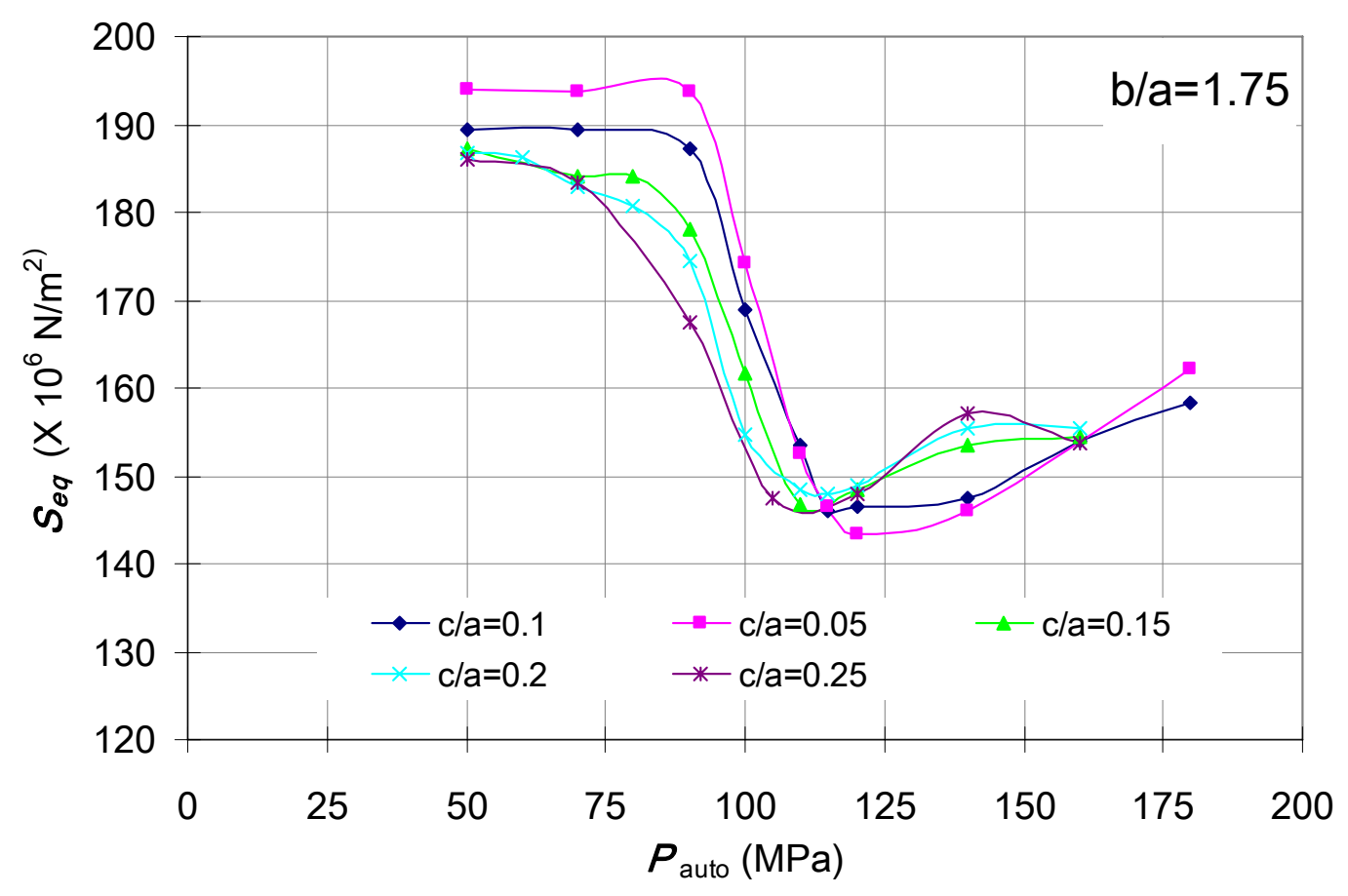

Figure 7 Variation of $\boldsymbol{S}_{\text {eq }}$ with $\boldsymbol{P}_{\text {auto }}$ for $b / a=1.75$ 


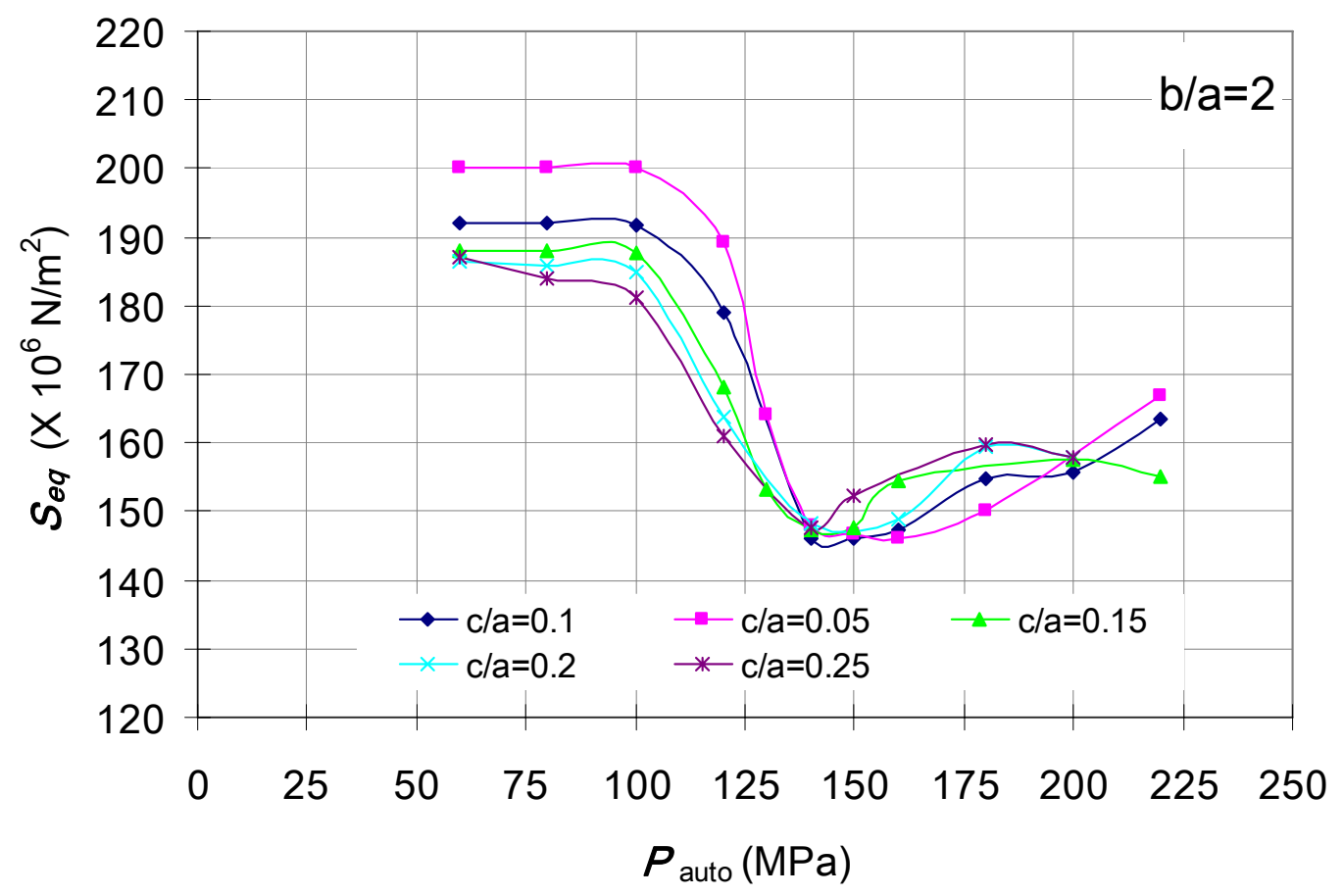

Figure 8 Variation of $\boldsymbol{S}_{\text {eq }}$ with $\boldsymbol{P}_{\text {auto }}$ for $b / a=2$

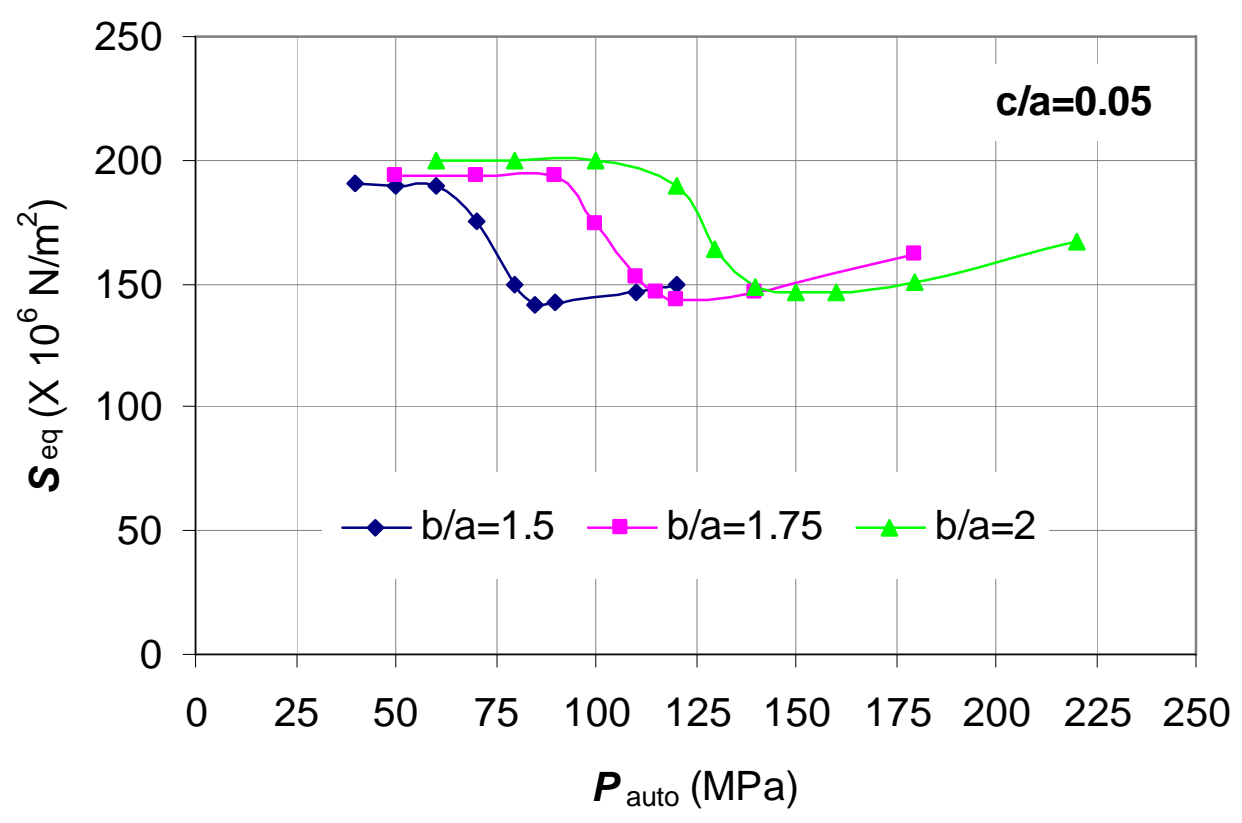

Figure 9 Variation of $\boldsymbol{S}_{\text {eq }}$ with $\boldsymbol{P}_{\text {auto }}$ for c/a $=0.05$ 


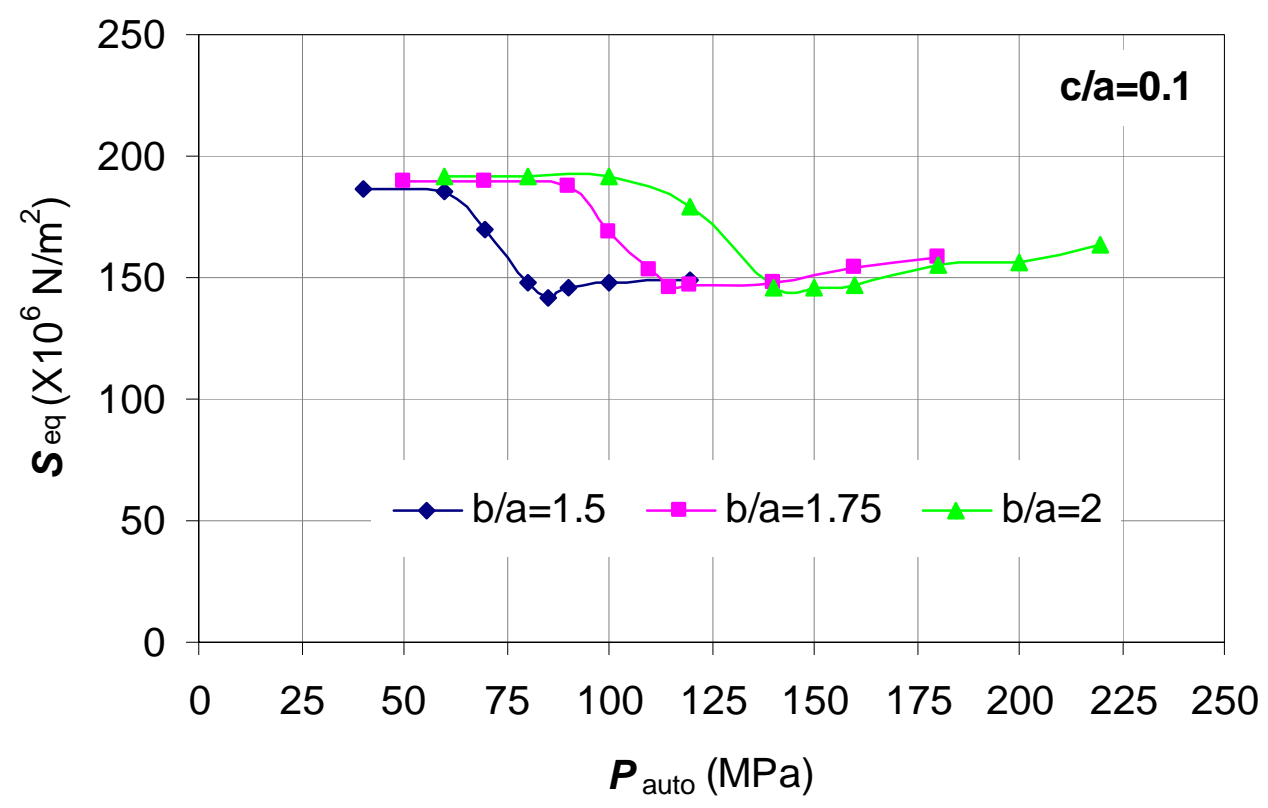

Figure 10 Variation of $\boldsymbol{S}_{\text {eq }}$ with $\boldsymbol{P}_{\text {auto }}$ for c/a $=0.1$

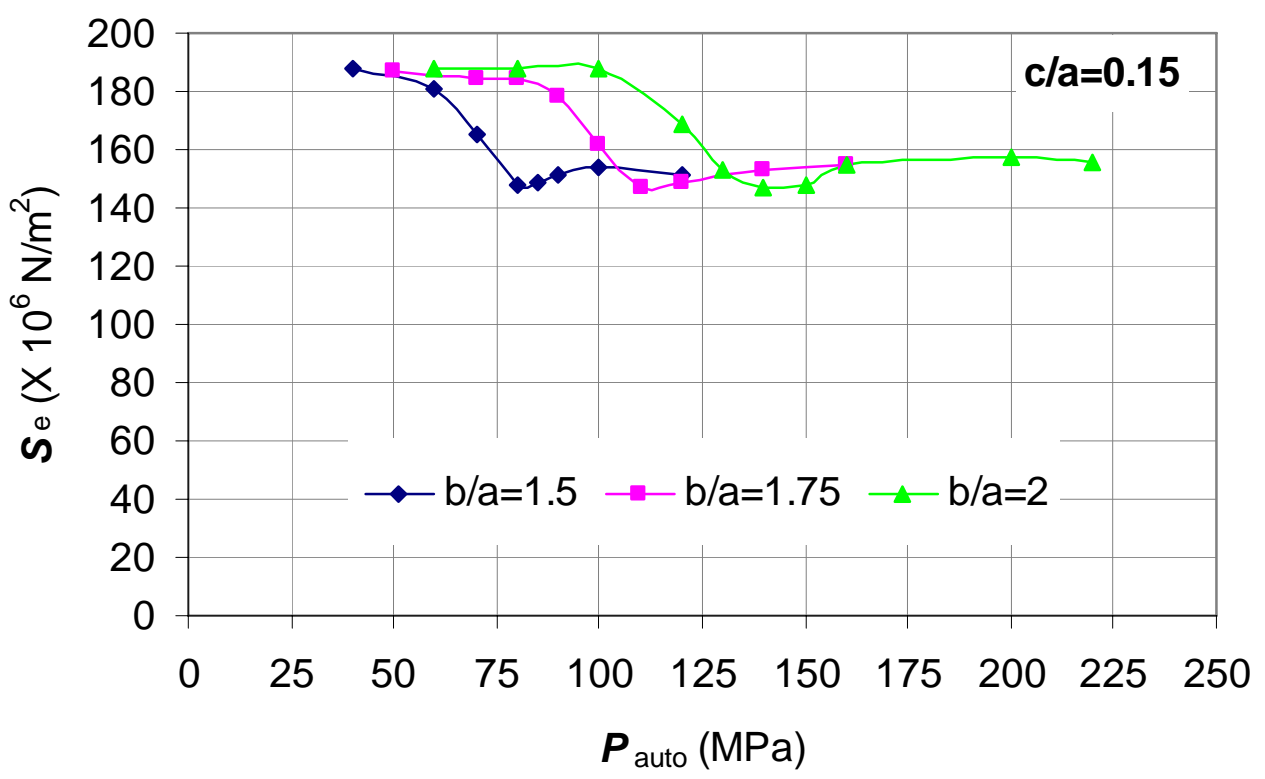

Figure 11 Variation of $\boldsymbol{S}_{\text {eq }}$ with $\boldsymbol{P}_{\text {auto }}$ for c/a $=0.15$ 


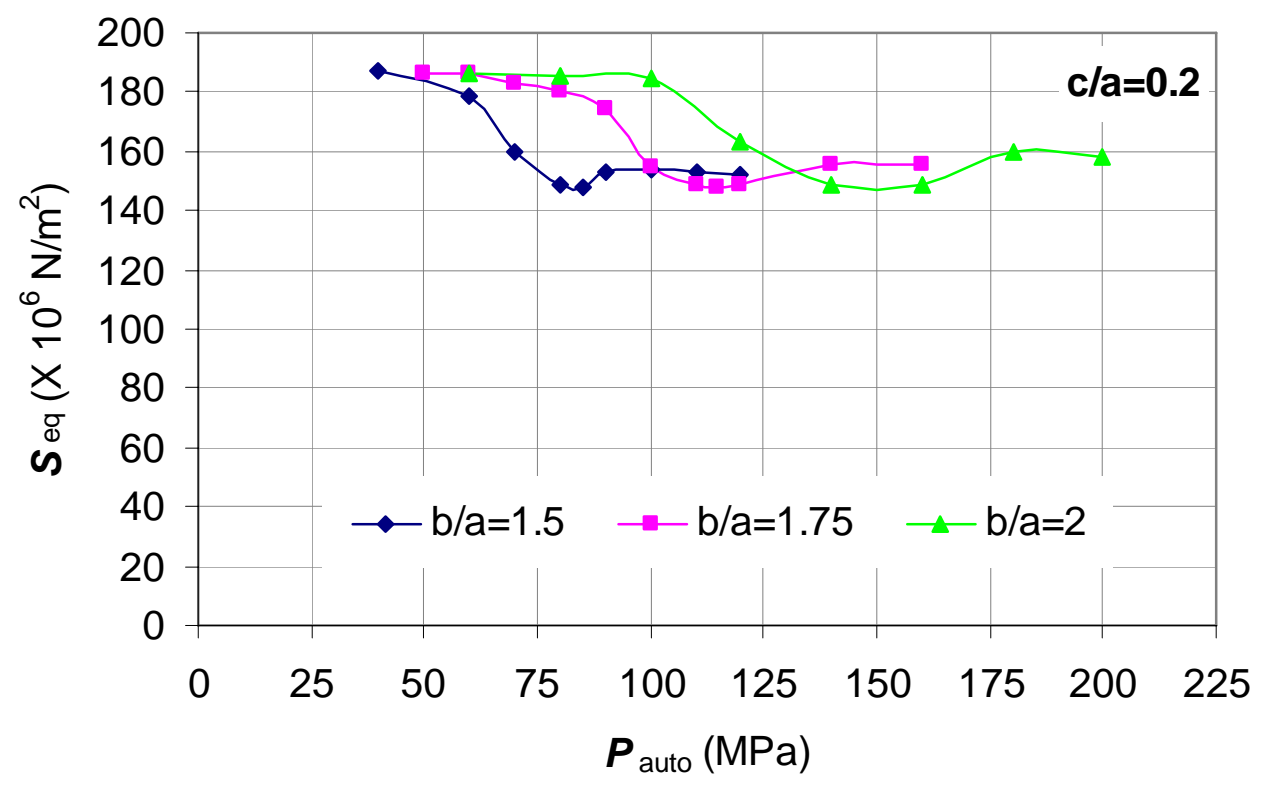

Figure 12 Variation of $\boldsymbol{S}_{\text {eq }}$ with $\boldsymbol{P}_{\text {auto }}$ for c/a $=0.2$

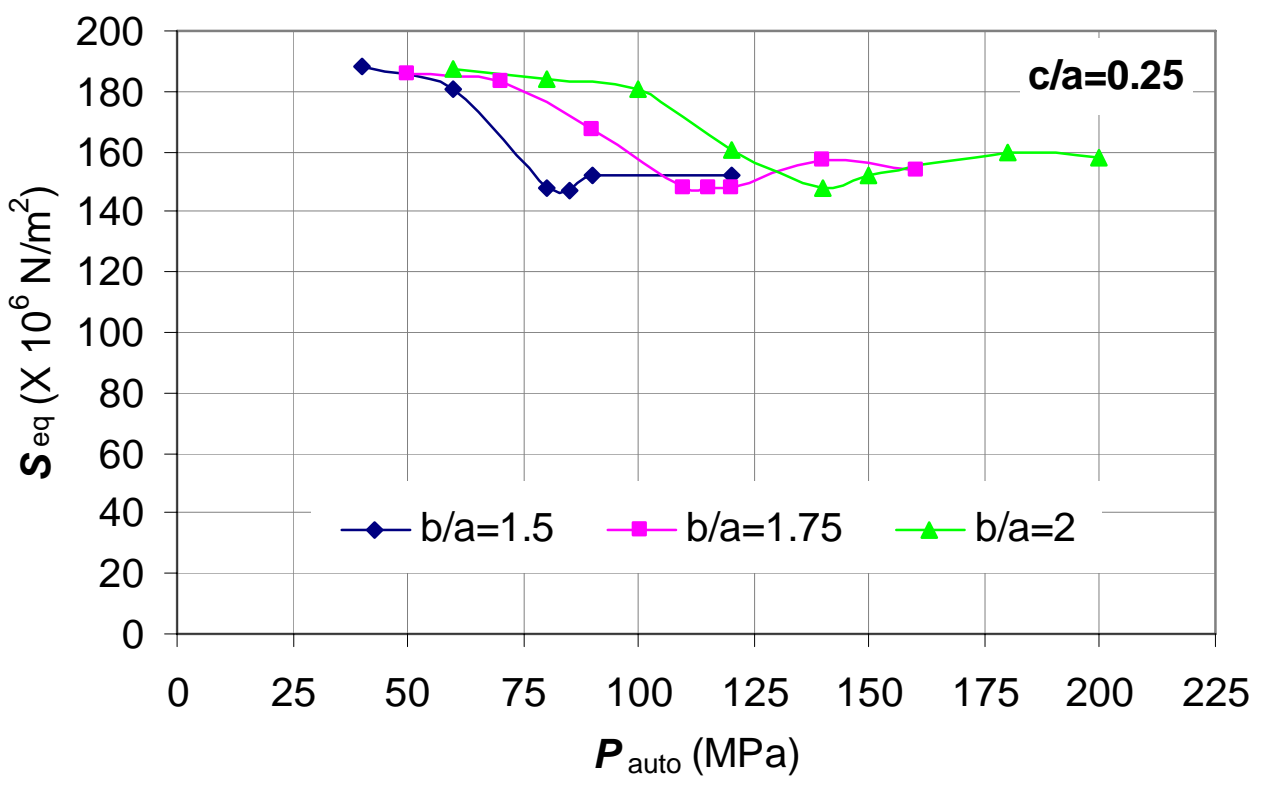

Figure 13 Variation of $\boldsymbol{S}_{\text {eq }}$ with $\boldsymbol{P}_{\text {auto }}$ for c/a $=0.25$ 


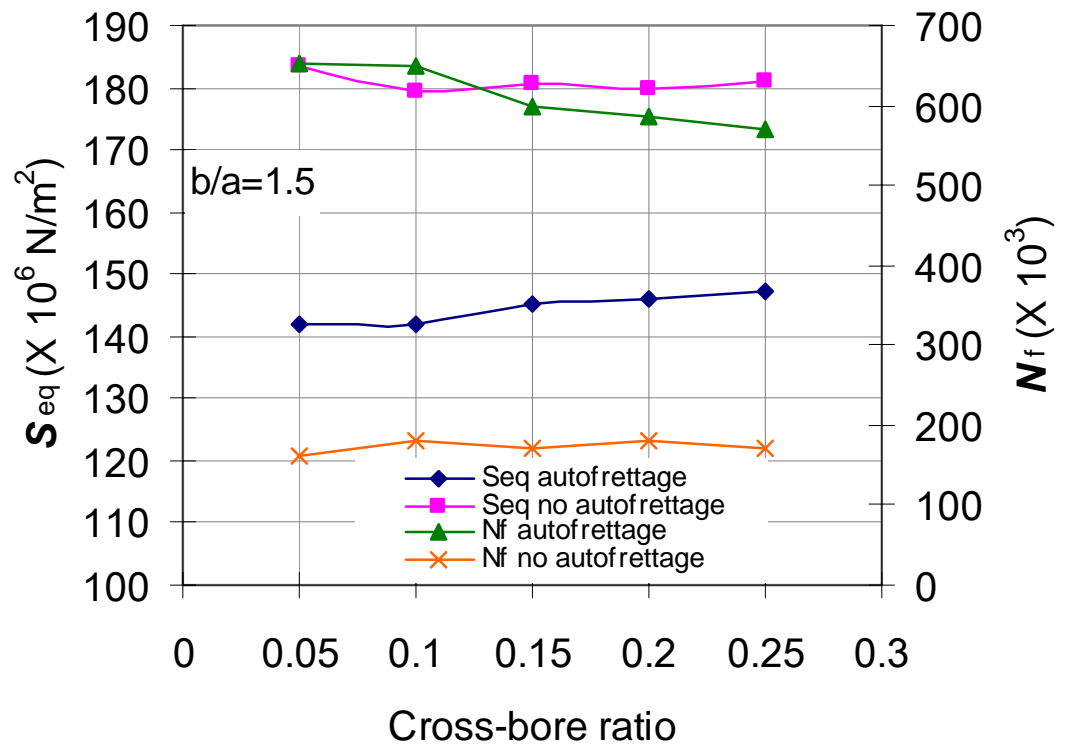

Figure 14 Fatigue life comparison for $\mathrm{b} / \mathrm{a}=1.5$

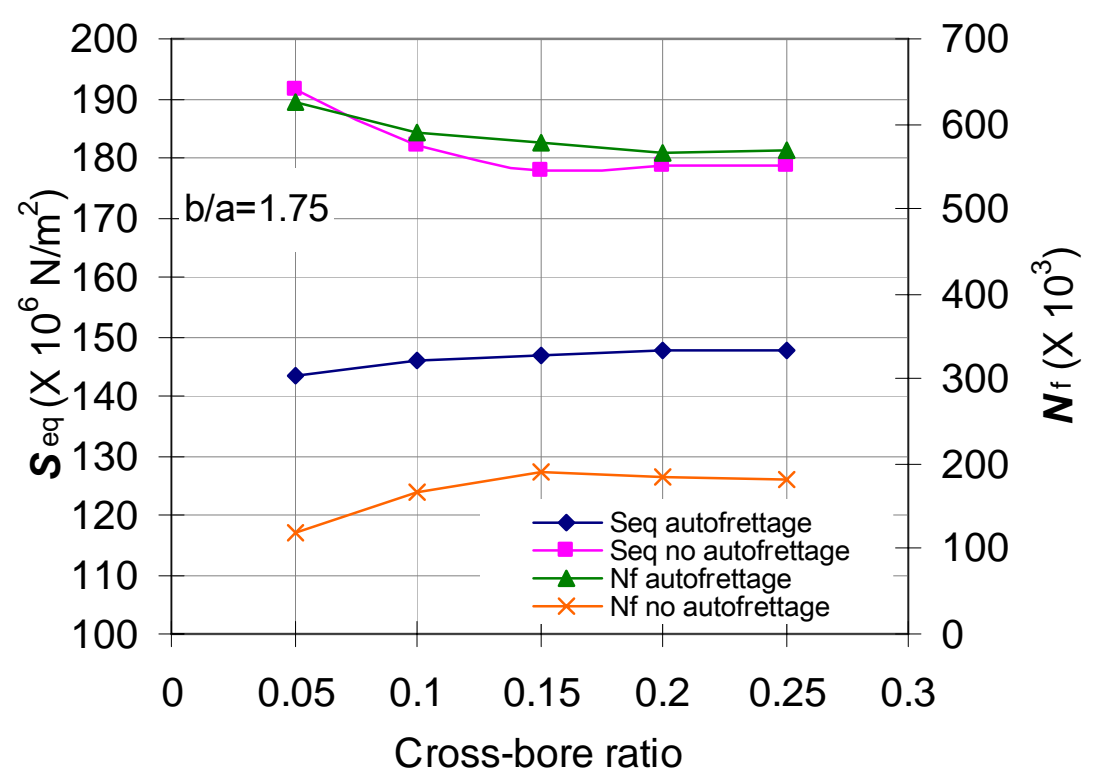

Figure 15 Fatigue life comparison for $\mathrm{b} / \mathrm{a}=1.75$ 


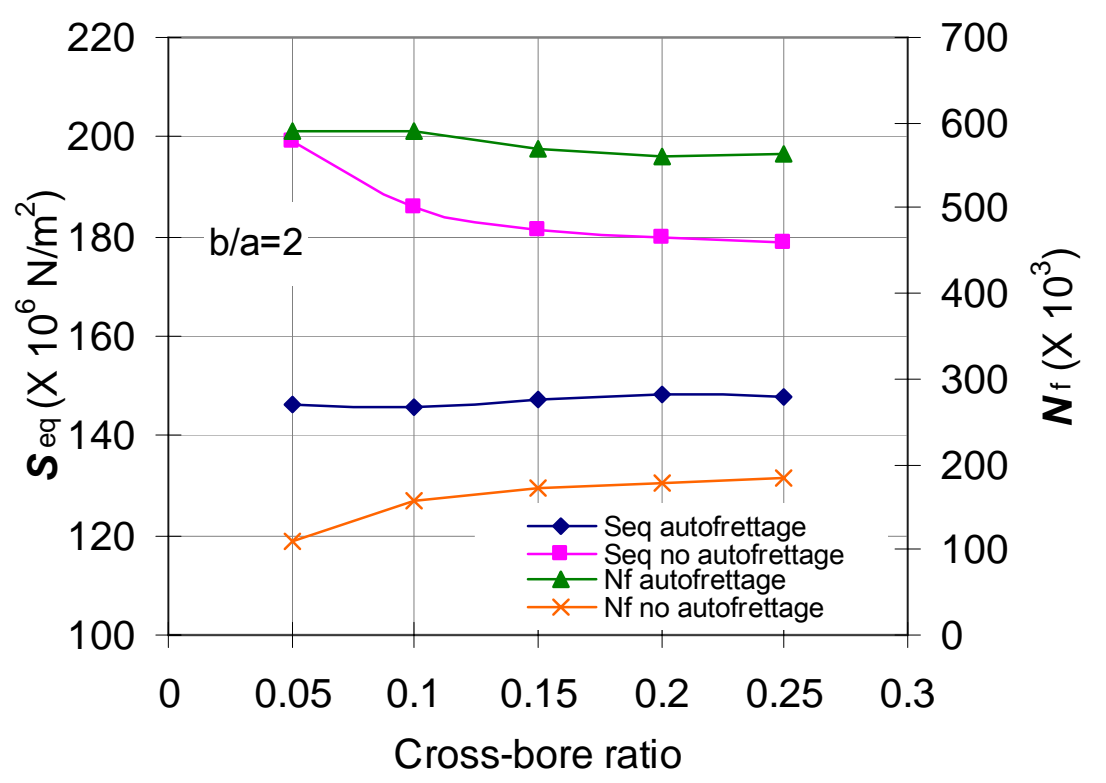

Figure 16 Fatigue life comparison for $\mathrm{b} / \mathrm{a}=2$ 


\section{Table}

\begin{tabular}{|c|c|c|c|c|c|c|}
\hline \multirow{2}{*}{$\begin{array}{c}\text { Cylinder radius } \\
\text { ratio } b / a\end{array}$} & & \multicolumn{5}{|c|}{ Cross-bore radius ratio, $c / a$} \\
\hline & & 0.05 & 0.1 & 0.15 & 0.2 & 0.25 \\
\hline 1.5 & \multirow{3}{*}{$\begin{array}{c}\text { Limit pressure } \\
\text { (MPa) }\end{array}$} & 136 & 133 & 129 & 125 & 122 \\
\hline 1.75 & & 189 & 188 & 180 & 176 & 171 \\
\hline 2 & & 234 & 232 & 227 & 220 & 216 \\
\hline 1.5 & \multirow{3}{*}{$\begin{array}{l}\text { First yield } \\
\text { pressure } \\
\text { (MPa) }\end{array}$} & 39 & 38 & 37 & 35 & 32 \\
\hline 1.75 & & 48 & 48 & 47 & 45 & 43 \\
\hline 2 & & 55 & 55 & 53 & 52 & 51 \\
\hline
\end{tabular}

Table 1 Limit and first yield pressures. 